\title{
Distribution Locational Marginal Pricing for Optimal Electric Vehicle Charging Management
}

\section{Li, Ruoyang; Wu, Qiuwei; Oren, Shmuel S.}

Published in:

IEEE Transactions on Power Systems

Link to article, DOI:

10.1109/TPWRS.2013.2278952

Publication date:

2013

Document Version

Early version, also known as pre-print

Link back to DTU Orbit

Citation (APA):

Li, R., Wu, Q., \& Oren, S. S. (2013). Distribution Locational Marginal Pricing for Optimal Electric Vehicle Charging Management. IEEE Transactions on Power Systems, 29(1), 203-211.

https://doi.org/10.1109/TPWRS.2013.2278952

\section{General rights}

Copyright and moral rights for the publications made accessible in the public portal are retained by the authors and/or other copyright owners and it is a condition of accessing publications that users recognise and abide by the legal requirements associated with these rights.

- Users may download and print one copy of any publication from the public portal for the purpose of private study or research.

- You may not further distribute the material or use it for any profit-making activity or commercial gain

- You may freely distribute the URL identifying the publication in the public portal

If you believe that this document breaches copyright please contact us providing details, and we will remove access to the work immediately and investigate your claim. 


\section{Distribution Locational Marginal Pricing for Optimal Electric Vehicle Charging Management}

\begin{tabular}{|r|l|}
\hline Journal: & IEEE Transactions on Power Systems \\
\hline Manuscript ID: & TPWRS-01388-2012.R3 \\
\hline Date Submitted by the Author: & 12-Aug-2013 \\
\hline Complete List of Authors: & $\begin{array}{l}\text { Li, Ruoyang; University of California at Berkeley, Department of Industrial } \\
\text { Engineering and Operations Research } \\
\text { Wu, Qiuwei; Technical University of Denmark, Department of Electrical } \\
\text { Engineering } \\
\text { Oren, Shmuel; University of California, }\end{array}$ \\
\hline Technical Topic Area : & Power system markets \\
\hline Key Words: & $\begin{array}{l}\text { Power system economics, Power system planning, Power distribution } \\
\text { planning }\end{array}$ \\
\hline \multicolumn{2}{|c}{} \\
\hline
\end{tabular}




\title{
Distribution Locational Marginal Pricing for Optimal Electric Vehicle Charging Management
}

\author{
Ruoyang Li, Student Member, IEEE, Qiuwei Wu, Member, IEEE, and Shmuel S. Oren, Fellow, IEEE
}

\begin{abstract}
This paper presents an integrated distribution locational marginal pricing (DLMP) method designed to alleviate congestion induced by electric vehicle (EV) loads in future power systems. In the proposed approach, the distribution system operator (DSO) determines distribution locational marginal prices (DLMPs) by solving the social welfare optimization of the electric distribution system which considers EV aggregators as price takers in the local DSO market and demand price elasticity. Nonlinear optimization has been used to solve the social welfare optimization problem in order to obtain the DLMPs. The efficacy of the proposed approach was demonstrated by using the bus 4 distribution system of the Roy Billinton Test System (RBTS) and Danish driving data. The case study results show that the integrated DLMP methodology can successfully alleviate the congestion caused by EV loads. It is also shown that the socially optimal charging schedule can be implemented through a decentralized mechanism where loads respond autonomously to the posted DLMPs by maximizing their individual net surplus.
\end{abstract}

Index Terms--Congestion management, distribution engineering, DLMP, DLMPs, DSO, EV, RBTS

\section{NOMENCLATURE}

$D_{l, i} \quad$ Power transfer distribution factor (PTDF) coefficient of line $l$ with respect to a unit injected at node $i$

$E_{i, t} \quad$ EV charging energy limit at time period $t$ at node $i$

$K_{l} \quad$ MVA capacity of line $l$

$N \quad$ Set of all nodes

$N_{c} \quad$ Subset of demand nodes

$N_{n} \quad$ Subset of non-demand nodes

$P_{D L M P, i, t} \quad$ Distribution locational marginal price at time period $t$ at node $i$ of the distribution grid

$P_{i, t}\left(\tau_{i}, t\right) \quad$ Benefits from using demand $\tau_{i}$ at time period $t$ at node $i$

$P_{L M P, t} \quad$ System locational marginal price (LMP) at time period $t$ for the node feeding the distribution grid

$S_{i, 0} \quad$ Initial aggregate battery state of charge (SOC) at node $i$

Ruoyang Li and Shmuel Oren are with Department of Industrial Engineering and Operations Research (IEOR), University of California at Berkeley (Email: ruoyang@berkeley.edu; oren@ieor.berkeley.edu).

Qiuwei Wu is with Center for Electric Power and Energy (CEE), Department of Electrical Engineering, Technical University of Denmark (DTU) (Email: qw@elektro.dtu.dk).
$S_{i, t}^{-} \quad$ Minimum aggregate battery SOC at time period $t$ at node $i$

$S_{i, t}^{+} \quad$ Maximum aggregate battery SOC at time period $t$ at node $i$

$T \quad$ Planning periods for optimization

$c_{i, t} \quad$ Conventional household demand at time period $t$ at node $i$

$g \quad$ The subset of generation node(s)

$p_{t} \quad$ Dual variables for total power flow balance constraints

$q_{g, t} \quad$ Generation supplied to the distribution grid at time period $t$

Net active power import/export at time period $t$ at generation node $g$ (positive for import)

Net active power import/ export at time period $t$ at node $i$ (positive for import)

EV charging energy at time period $t$ at node $i$ $x_{i, t}$

$\kappa_{i, t}^{-}$

$\kappa_{i, t}^{+}$

$\lambda_{l, t}^{-}$

$\lambda_{l, t}^{+}$

$\mu_{i, t}^{-}$

$\mu_{i, t}^{+}$

$\xi_{i, t}$

$\rho_{i, t}$

$\tau_{i, t}$

$\omega_{g, t}$

$\omega_{i, t}$
Dual variables for aggregate EV minimum SOC constraints

Dual variables for aggregate EV maximum SOC constraints

Dual variables for negative line flow constraints

Dual variables for positive line flow constraints

Dual variables for EV minimum charging energy constraints

Dual variables for EV maximum charging energy constraints

Dual variables for conventional household demand constraints

Dual variables for demand node power balance constraints

Demand variables at time period $t$ at node $i$

Dual variables for generation node power balance constraints

Dual variables for non-demand node net active power import/output constraints

\section{INTRODUCTION}

$\mathrm{E}$ nvironmental concerns and the quest for energy supply independence have resulted in increasing penetration of renewable energy sources (RES) and a move toward 
electrification of transportation. Consequently, electric vehicles (EVs) are expected to play a significant role in the future power systems and distribution networks. Increased use of EVs will reduce the green house gas (GHG) emission from the transport sector by replacing conventional internal combustion engine (ICE) vehicles while also serving as distributed energy storage that can mitigate uncertainties arising from intermittent RES.

Numerous studies have addressed vehicle-to-grid (V2G) technology to investigate the technical and commercial feasibility of providing ancillary service to the grid from EVs. The capacity from EVs and the economic return to participate in peak power, spinning reserve and regulation markets have been explored in [1]-[3]. The effectiveness of using EVs to provide peak load shaving and extra flexibility has been illustrated in [4] and [5].

However, the deployment of a large number of EVs will challenge power system operations especially for distribution networks if there is no proper coordination of the EV charging. Grid congestion results from demand patterns that induce flows exceeding design limits. Congestion from EVs can be observed at the medium voltage (MV) level, as demonstrated by a number of studies [6]-[9]. It was also noted that the problems are likely to originate on the distribution network, and as such, analysis of these networks should be conducted as the primary stage of EV induced congestion [9]-[11].

Grid congestion depends on a number of factors including local grid rating and topology, penetration and distribution of EVs, and charging management procedures. Coordinated charging appears to be an effective means of allowing increased penetration of EVs without violating grid constraints. There is some diversity regarding the optimal manner in which to coordinate charging and the proposed objectives for such coordination include minimization of losses [7], maximization of EV penetration [9], and minimization of customer charging costs [12]-[13].

The congestion management methods can be categorized into three groups: Optimal Power Flow (OPF) based method, price area congestion control method and transaction-based methods [14]. The OPF based congestion management method is based on a centralized optimization and is considered to be the most accurate and effective congestion management method. Price-based congestion management controls congestion by generation re-dispatch in response to congestion prices within an OPF framework [15].

In the existing work on load management techniques and other methods for alleviating congestions from EVs, there is no integrated method which has a closed loop solution accounting for conventional demand elasticity and EV demand shifting characteristics. In order to address this problem, the distribution locational marginal pricing (DLMP) method is proposed for electric distribution networks in order to alleviate congestion induced by EVs. In the proposed method, the distribution system operator (DSO) determines the distribution locational marginal prices (DLMPs) by solving the social welfare optimization for the electric distribution network which considers EV aggregators as price takers in the local DSO market and demand elasticity for residential energy consumption. It is assumed that all the EV aggregators are economically rational, i.e. their objective is to maximize their individual surplus.

The paper is arranged as follows. The mathematical formulation of the integrated DLMP method and the determination of DLMPs are presented in Section III. In Section IV, the EV aggregator based optimal charging management is described. The alleviation of congestion induced by EVs within electric distribution networks is explained in Section V. Case studies were conducted using the bus 4 distribution networks of the Roy Billinton Test System (RBTS) [16] and the Danish driving data, and the case study results are presented in Section VI with detailed discussion followed by the conclusion section.

\section{DETERMINATION OF DistribUtion LOCATIONAL MAR- GINAL PRICES USING INTEGRATED OPTIMIZATION}

The system LMPs are determined by minimizing the cost of generations with the physical constraints of the transmission system respected, which exposes producers and consumers to the marginal cost of electricity delivery at different locations. The LMPs can be decomposed into three components: marginal cost of generation, marginal cost of losses and marginal cost of congestion [17].

The LMPs can be computed by either AC optimal power flow (ACOPF) or DC optimal power flow (DCOPF). The DCOPF is widely used and is considered to be sufficient for LMP calculation due to its computational efficiency and approximation accuracy [18]. The DCOPF has also been employed by several software tools for chronological LMP simulation and forecasting, such as ABB GridViewTM, Siemens Promod, GE MAPSTM and PowerWorld [19].

The DCOPF was adopted in the derivation of DLMPs as a practical approach to address the computational complexity resulting from the large number of nodes within the electric distribution network. In the proposed DLMP algorithm, the DSO determines the DLMPs for the next day by solving a constrained social welfare maximization problem.

The mathematical formulation in [20]-[22] has been modified to make it more general to allow economic allocation for both conventional household demand and EV charging energy. The mathematical formulation of the DSO optimization problem is presented in (1) to (9),

Objective Function

$$
\max \sum_{i \in N_{c}} \sum_{t \in T} \int_{0}^{c_{i, t}} P_{i, t}\left(\tau_{i}, t\right) d \tau_{i, t}-\sum_{t \in T} P_{L M P, t} q_{g, t}
$$

subject to

$$
\begin{array}{rlr}
\sum_{i \in N} r_{i, t}=0 & \forall t \in T & \left(p_{t}\right)(2) \\
-K_{l} \leq \sum_{i \in N} D_{l, i} r_{i, t} \leq K_{l} & \forall l \in L, \forall t \in T & \left(\lambda_{l, t}^{-}, \lambda_{l, t}^{+}\right)(3) \\
r_{i, t}=0 & \forall i \in N_{n}, \forall t \in T & \left(\omega_{i, t}\right)(4) \\
r_{g, t}+q_{g, t}=0 & \forall t \in T & \left(\omega_{g, t}\right)(5) \\
r_{i, t}=c_{i, t}+x_{i, t} & \forall i \in N_{c}, \forall t \in T & \left(\rho_{i, t}\right)(6) \\
c_{i, t} \geq 0 & \forall i \in N_{c}, \forall t \in T & \left(\xi_{i, t}\right)(7) \\
0 \leq x_{i, t} \leq E_{i, t} & \forall i \in N_{c}, \forall t \in T & \left(\mu_{i, t}^{-}, \mu_{i, t}^{+}\right)(8) \\
S_{i, t}^{-} \leq S_{i, 0}+\sum_{t^{\prime} \leq t-1} x_{i, t^{\prime}}-\sum_{t^{\prime} \leq t} d_{i, t^{\prime}} \leq S_{i, t}^{+} & \forall i \in N_{c}, \forall t \in T \backslash\{1\} & \left(\kappa_{i, t}^{-}, \kappa_{i, t}^{+}\right)
\end{array}
$$


The DSO objective is to maximize the social surplus in (1) subject to the energy-balance constraints in (2), the transmission constraints in (3), the non-demand node constraints in (4), generation node balance constraints in (5), the demand node balance constraints in (6), the conventional household demand non-negativity constraints in (7), the charging energy limit constraints in (8) and the driving requirement constraints in (9).

For the demand node balance constraints in (6), the assumption is that EVs only charge energy at the location they belong to, which requires that the energy import $r_{i, t}$ is the sum of the conventional household demand $c_{i, t}$ and EV demand $x_{i, t}$ at time period $t$ at node $i$. The elastic conventional household demand $c_{i, t}$ is constrained to be non-negative in (7). The EV demand $x_{i, t}$ is constrained between 0 and charging energy limit $E_{i, t}$ at time period $t$ at node $i$ in (8). $E_{i, t}$ varies over time to reflect the availability of EVs across hours. The SOC of EV batteries at time period $t$ at node $i$ is the sum of its initial SOC and total charging energy $x_{i, t}$ up to time period $t-1$ minus the total driving energy requirement $d_{i, t}$ up to time period $t$. The SOC is constrained between minimum SOC $S_{i, t}^{-}$and maximum SOC $S_{i, t}^{+}$in (9). The variables in parentheses next to each constraint denote the Lagrange multipliers corresponding to that constraint.

The objective function consists of two components, social value of meeting the conventional demand, given by the area under the demand functions, and the cost of satisfying both the $\mathrm{EV}$ demand and the conventional demand as shown in (1). The benefit of the EV demand is not included in the objective function since that component is constant, as long as the EV demand is met within the day, and is not affected by the charging schedule. Instead, a constraint requiring that the EV demand be met by the schedule is included. To be more specific, the object function in (1) can be further decomposed into three terms as shown in (10),

$$
\begin{aligned}
& \sum_{i \in N_{c}} \sum_{t \in T} \int_{0}^{c_{i, t}} P_{i, t}\left(\tau_{i}, t\right) d \tau_{i, t}-\sum_{t \in T} P_{L M P, t} q_{g, t} \\
& =\sum_{i \in N_{c}} \sum_{t \in T} \int_{0}^{c_{i, t}} P_{i, t}\left(\tau_{i}, t\right) d \tau_{i, t}-\sum_{t \in T} P_{L M P, t} \sum_{i \in N_{c}}\left(c_{i, t}+x_{i, t}\right) \\
& =\sum_{i \in N_{c}} \sum_{t \in T} \int_{0}^{c_{i, t}} P_{i, t}\left(\tau_{i}, t\right) d \tau_{i, t}-\sum_{t \in T} P_{L M P, t} \sum_{i \in N_{c}} c_{i, t}-\sum_{t \in T} P_{L M P, t} \sum_{i \in N_{c}} x_{i, t}
\end{aligned}
$$

where $\sum_{i \in N_{c}} \sum_{t \in T} \int_{0}^{c_{i, t}} P_{i, t}\left(\tau_{i}, t\right) d \tau_{i, t}-\sum_{t \in T} P_{L M P, t} \sum_{i \in N_{c}} c_{i, t}$ is the social welfare corresponding to the conventional demand and $\sum_{t \in T} P_{L M P, t} \sum_{i \in N_{c}} x_{i, t}$ is the EV charging cost.

The Karush-Kuhn-Tucker (KKT) optimality conditions for the social welfare optimization problem are summarized in (11) to (28),

$$
\begin{aligned}
P_{i, t}\left(c_{i, t}\right)-\rho_{i, t}+\xi_{i, t}=0 & \forall i \in N_{c}, \forall t \in T \\
-p_{t}-\sum_{l \in L}\left(\lambda_{l, t}^{+}-\lambda_{l, t}^{-}\right) D_{l, i}+\rho_{i, t}=0 & \forall i \in N_{c}, \forall t \in T
\end{aligned}
$$

$$
\begin{aligned}
-p_{t}-\sum_{l \in L}\left(\lambda_{l, t}^{+}-\lambda_{l, t}^{-}\right) D_{l, i}+\omega_{i, t}=0 & \forall i \in N_{n}, \forall t \in T \\
-p_{t}-\sum_{l \in L}\left(\lambda_{l, t}^{+}-\lambda_{l, t}^{-}\right) D_{l, g}+\omega_{g, t}=0 & \forall t \in T \\
-P_{L M P, t}+\omega_{g, t}=0 & \forall t \in T \\
-\rho_{i, t}-\left(\mu_{i, t}^{+}-\mu_{i, t}^{-}\right)-\sum_{t^{\prime} \geq t+1}\left(\kappa_{i, t^{\prime}}^{+}-\kappa_{i, t^{\prime}}^{-}\right)=0 & \forall i \in N_{c}, \forall t \in T \backslash\{|T|\} \\
-\rho_{i, t}-\left(\mu_{i, t}^{+}-\mu_{i, t}^{-}\right)=0 & \forall i \in N_{c}, \forall t=|T| \\
\sum_{i \in N} r_{i, t}=0 & \forall t \in T \\
r_{i, t}=0 & \forall i \in N_{n}, \forall t \in T \\
r_{g, t}+q_{g, t}=0 & \forall t \in T \\
r_{i, t}=c_{i, t}+x_{i, t} & \forall i \in N_{c}, \forall t \in T \\
\lambda_{l, t}^{-} \geq 0 \perp \sum_{i \in N} D_{l, i} r_{i, t}+K_{l} \geq 0 & \forall l \in L, \forall t \in T \\
\lambda_{l, t}^{+} \geq 0 \perp K_{l}-\sum_{i \in N} D_{l, i} r_{i, t} \geq 0 & \forall l \in L, \forall t \in T \\
\xi_{i, t} \geq 0 \perp c_{i, t} \geq 0 & \forall i \in N_{c}, \forall t \in T \\
\mu_{i, t}^{-} \geq 0 \perp x_{i, t} \geq 0 & \forall i \in N_{c}, \forall t \in T \\
\mu_{i, t}^{+} \geq 0 \perp E_{i, t}-x_{i, t} \geq 0 & \forall i \in N_{c}, \forall t \in T
\end{aligned}
$$

The KKT conditions yield the optimality for the primal problem and provide an economic interpretation of the Lagrange multipliers. The DLMPs are derived from the KKT conditions to provide price incentives for market participants to alleviate congestion and ensure efficient load allocation. By solving (12), (14) and (15), the marginal value of a unit of EV charging energy or conventional demand at time period $t$ at node $i, \rho_{i, t}$, takes the form in (29),

$$
\rho_{i, t}=P_{L M P, t}-\sum_{l \in L}\left(\lambda_{l, t}^{+}-\lambda_{l, t}^{-}\right) D_{l, g}+\sum_{l \in L}\left(\lambda_{l, t}^{+}-\lambda_{l, t}^{-}\right) D_{l, i}
$$

In the RBTS, the power transfer distribution factor (PTDF) coefficient associated with the generation node $D_{l, g}$ is set to be 0 to enable unlimited import from the grid to the distribution network, which simplifies (29) and yields (30),

$$
\rho_{i, t}=P_{L M P, t}+\sum_{l \in L}\left(\lambda_{l, t}^{+}-\lambda_{l, t}^{-}\right) D_{l, i}
$$

The DLMPs can be derived by combining (11) and (30),

$$
\begin{aligned}
P_{D L M P, i, t} & =P_{i, t}\left(c_{i, t}\right)=\rho_{i, t}-\xi_{i, t} \\
& =P_{L M P, t}+\sum_{l \in L}\left(\lambda_{l, t}^{+}-\lambda_{l, t}^{-}\right) D_{l, i}-\xi_{i, t}
\end{aligned}
$$

The non-negativity constraint (7) can be excluded by implicitly assuming an interior solution with respect to these constraints, forcing the dual variable associated with the constraint $\xi_{i, t}=0$. This can be explained as: every conventional household consumes at least a small positive amount of energy. Under this assumption, the DLMPs become,

$$
P_{D L M P, i, t}=P_{L M P, t}+\varphi_{i, t}
$$


where $\varphi_{i, t}=\sum_{l \in L}\left(\lambda_{l, t}^{+}-\lambda_{l, t}^{-}\right) D_{l, i}$. The DLMPs can be interpreted as the sum of the reference price $P_{L M P, t}$ and the locational congestion markup $\varphi_{i, t}$, which is analogous to the marginal cost of congestion in the LMPs.

Noticing that the LMPs only optimize the dispatch of instantaneous demand, the DLMPs are designed to co-optimize the dispatch of both the instantaneous demand and the aggregated EV charging schedule over the planning interval. By rearranging (16) and (17), $\rho_{i, t}$ can be written as (34),

$$
\rho_{i, t}=\left\{\begin{array}{rr}
-\mu_{i, t}^{+}+\mu_{i, t}^{-}-\sum_{t^{\prime} \geq t+1} \kappa_{i, t^{\prime}}^{+}+\sum_{t^{\prime} \geq t+1} \kappa_{i, t^{\prime}}^{-} & \forall i \in N_{c}, \forall t \in T \backslash\{|T|\} \\
-\mu_{i, t}^{+}+\mu_{i, t}^{-} & \forall i \in N_{c}, \forall t=|T|
\end{array}\right.
$$

where $-\mu_{i, t}^{+}+\mu_{i, t}^{-}-\sum_{t^{\prime} \geq t+1} \kappa_{i, t^{\prime}}^{+}+\sum_{t^{\prime} \geq t+1} \kappa_{i, t^{\prime}}^{-}$is the marginal value of energy at non-terminal period $t \in T \backslash\{|T|\}$ at node $i$, and $-\mu_{i, t}^{+}+\mu_{i, t}^{-}$is marginal value of energy at terminal period $t=|T|$ at node $i$. Combining (11) and (34) gives the DLMPs at time period $t$ at node $i$ as a linear combination of the dual variables associated with constraints of EVs,

$$
\begin{gathered}
P_{\text {DLMP }, i, t}=P_{i, t}\left(c_{i, t}\right)=\rho_{i, t}-\xi_{i, t} \\
=\left\{\begin{array}{cc}
-\mu_{i, t}^{+}+\mu_{i, t}^{-}-\sum_{t^{\prime} \geq t+1} \kappa_{i, t^{\prime}}^{+}+\sum_{t^{\prime} \geq t+1} \kappa_{i, t^{\prime}}^{-}-\xi_{i, t} & \forall i \in N_{c}, \forall t \in T \backslash\{|T|\} \\
-\mu_{i, t}^{+}+\mu_{i, t}^{-}-\xi_{i, t} & \forall i \in N_{c}, \forall t=|T|
\end{array}\right. \\
=\left\{\begin{array}{cc}
-\mu_{i, t}^{+}+\mu_{i, t}^{-}-\sum_{t^{\prime} \geq t+1} \kappa_{i, t^{\prime}}^{+}+\sum_{t^{\prime} \geq t+1} \kappa_{i, t^{\prime}}^{-} & \forall i \in N_{c}, \forall t \in T \backslash\{|T|\} \\
-\mu_{i, t}^{+}+\mu_{i, t}^{-} & \forall i \in N_{c}, \forall t=|T|
\end{array}\right.
\end{gathered}
$$

where $\xi_{i, t}=0$ assuming (7) does not bind.

The DLMPs defined by (33) and (37) can be interpreted as the equilibrium conditions for the electric distribution system market clearing. The market dynamics and the economic behavior of market participants under the DLMPs are discussed in the Section IV.

\section{Aggregator based Optimal EV Charging MANAGE- MENT}

The EV charging management can take different forms: charging management controlled by individual EV users, aggregator based charging management and proper mixture of the two mechanisms. In this paper, the aggregator based EV charging management implementation is used.

In the aggregator based EV charging management concept, the EV aggregator is a profit-seeking entity, who takes care of the EV fleet on behalf of the EV users, ensures that the energy needs are satisfied, and provides customized service and charging solution. The objective of EV aggregators is to meet the energy needs of EV users with the minimum charging cost. It is also assumed that each EV aggregator only controls a small portion of the EVs so that EV aggregators do not have market power and act as price takers in the DSO market. The aggregator based EV optimal charging management can be described by the optimization problem in (38) to (40), Objective Function subject to

$$
\min \sum_{t \in T} P_{D L M P, i, t} x_{i, t}
$$

$$
\begin{aligned}
0 \leq x_{i, t} \leq E_{i, t} \quad \forall t \in T \quad\left(\mu_{i, t}^{-}, \mu_{i, t}^{+}\right) \\
S_{i, t}^{-} \leq S_{i, 0}+\sum_{t^{\prime} \leq t-1} x_{i, t^{\prime}}-\sum_{t^{\prime} \leq t} d_{i, t^{\prime}} \leq S_{i, t}^{+} \quad \forall t \in T \backslash\{1\}\left(\kappa_{i, t}^{-}, \kappa_{i, t}^{+}\right)
\end{aligned}
$$

The constraints in (39) and (40) are to ensure that the EV charging energy and the EV battery SOC are within the specified limits. When the DLMPs, $P_{D L M P, i, t}$, are known to the EV aggregator, the optimization problem is a linear programming problem and the EV aggregator optimally decides $x_{i, t}$, the amount of energy to purchase in each hour, to minimize the charging cost subject to the charging power limit constraints and the driving requirement constraints. The optimality conditions of the EV charging are summarized in (41) to (52),

$$
\begin{aligned}
& 0 \leq x_{i, t} \leq E_{i, t} \quad \forall t \in T \\
& S_{i, t}^{-} \leq S_{i, 0}+\sum_{t^{\prime} \leq t-1} x_{i, t^{\prime}}-\sum_{t^{\prime} \leq t} d_{i, t^{\prime}} \leq S_{i, t}^{+} \quad \forall t \in T \backslash\{1\} \\
& -P_{D L M P, i, t}-\mu_{i, t}^{+}+\mu_{i, t}^{-}-\sum_{t^{\prime} \geq t+1} \kappa_{i, t^{\prime}}^{+}+\sum_{t^{\prime} \geq t+1} \kappa_{i, t^{\prime}}^{-}=0 \quad \forall t \in T \backslash\{|T|\} \\
& -P_{D L M P, i, t}-\mu_{i . t}^{+}+\mu_{i, t}^{-}=0 \quad \forall t=|T| \\
& \mu_{i, t}^{-} \geq 0 \quad \forall t \in T \\
& \mu_{i . t}^{+} \geq 0 \quad \forall t \in T \\
& \kappa_{i, t}^{-} \geq 0 \quad \forall t \in T \backslash\{1\} \\
& \kappa_{i, t}^{+} \geq 0 \quad \forall t \in T \backslash\{1\} \\
& \mu_{i, t}^{-} x_{i, t}=0 \quad \forall t \in T \\
& \mu_{i, t}^{+}\left(E_{i, t}-x_{i, t}\right)=0 \quad \forall t \in T \\
& \kappa_{i, t}^{-}\left(S_{i, 0}+\sum_{t^{\prime} \leq t-1} x_{i, t^{\prime}}-\sum_{t^{\prime} \leq t} d_{i, t^{\prime}}-S_{i, t}^{-}\right)=0 \quad \forall t \in T \backslash\{1\} \\
& \kappa_{i, t}^{+}\left(S_{i, t}^{+}-S_{i, 0}-\sum_{t^{\prime} \leq t-1} x_{i, t^{\prime}}+\sum_{t^{\prime} \leq t} d_{i, t^{\prime}}\right)=0 \quad \forall t \in T \backslash\{1\}
\end{aligned}
$$

(41)-(42) are the primal feasibility conditions. (43)-(48) are the dual feasibility conditions. (49)-(52) are the complementarity conditions.

Theorem 1 The efficient allocation of EV charging of the DSO problem $x_{i, t}^{*}$ is optimal for each EV aggregator under the DLMPs, if the non-negativity constraint of conventional household demand (7) does not bind.

Proof: It has been shown that the optimal solution of the DSO problem $\left\{x_{i, t}^{*}, \mu_{i, t}^{+*}, \mu_{i, t}^{-*}, \kappa_{i, t}^{+*}, \kappa_{i, t}^{-*}\right\}$ also satisfies the optimality conditions of the EV aggregator's problem in (41)-(52).

The optimal solution of the DSO problem satisfies the KKT conditions (11)-(28). If (7) does not bind, the optimal solution of the DSO problem satisfies (37),

$$
P_{D L M P, i, t}^{*}=\left\{\begin{array}{rr}
-\mu_{i, t}^{+*}+\mu_{i, t}^{-*}-\sum_{t^{\prime} \geq t+1} \kappa_{i, t^{+*}}^{+*}+\sum_{t^{\prime} \geq t+1} \kappa_{i, t^{\prime}}^{-*} & \forall t \in T \backslash\{|T|\} \\
-\mu_{i, t}^{*}+\mu_{i, t}^{-*} & \forall t=|T|
\end{array}\right.
$$

This implies (43) and (44) hold under the optimal solution $\left\{x_{i, t}^{*}, \mu_{i, t}^{+^{*}}, \mu_{i, t}^{-*}, \kappa_{i, t}^{+*}, \kappa_{i, t}^{-*}\right\}$. (41), (42) and (45)-(52) come directly from KKT conditions (25)-(28). Thus, the efficient allocation of EV charging from the DSO problem satisfies the optimality conditions of the EV aggregator's problem. 
Corollary 1 The efficient allocation of the DSO problem $\left\{x_{i, t}^{*}, c_{i, t}^{*}\right\}$ can be achieved in a decentralized system under the DLMPs, if the non-negativity constraint of conventional household demand (7) does not bind.

Proof: The conventional household demand $c_{i, t}^{*}$ is deterministic under the DLMPs. From Theorem 1, it is known that, under the DLMPs, the optimal solution of the EV aggregator's problem is the efficient allocation of EV charging of the DSO problem $x_{i, t}^{*}$. Therefore, the efficient allocation of the DSO problem can be achieved in the decentralized implementation.

\section{ALLEVIATING CONGESTION FROM EVS WITHIN ELECTRIC DISTRIBUTION NETWORKS USING DLMP}

The intention of the proposed DLMP concept is to alleviate congestion within electric distribution networks which might be caused by the EV charging demand. The congestion alleviation approach using DLMP is illustrated in Fig. 1.

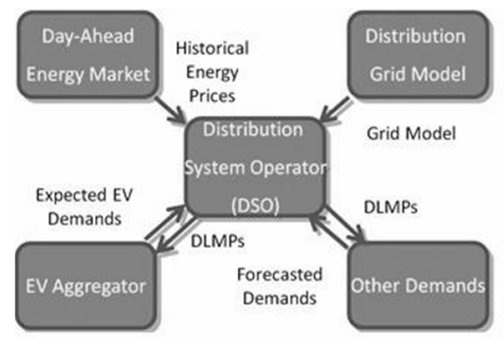

Fig. 1. Congestion Alleviation from EVs using DLMPs

The DSO plays a major role in the DLMP based congestion management within electric distribution networks. The concept can be explained by the following steps.

- The DSO obtains the LMPs from the posted dayahead energy prices.

- According to the EV data within the electric distribution network, the expected EV demand will be forecasted by the DSO with the assumption that all EV aggregators are minimizing their EV charging costs. Conventional demand will be forecasted by the DSO according to the posted energy prices.

- With the information on the forecasted demand, the DSO calculates the DLMPs at the electric distribution network level taking into account the electric distribution network topology.

- In the end, the DLMPs will be sent to all EV aggregators and retailers.

As it is proved in Theorem 1 and Corollary 1, after receiving the DLMPs from the DSO, EV aggregators and retailers will behave exactly as the DSO predicts. Consequently, the congestion on the electric distribution network will be properly managed, while it only requires EV aggregators and retailers to react rationally to the DLMPs by maximizing their individual net surplus. At this point, any additional information of distribution network grid or line congestion is redundant to the decision-making process of $\mathrm{EV}$ aggregators and retailers.

\section{CASE STUdiEs}

In order to illustrate the efficacy of the proposed DLMP concept in alleviating congestion from EV demand, case studies have been conducted using the bus 4 distribution network of the RBTS with the Danish driving data.

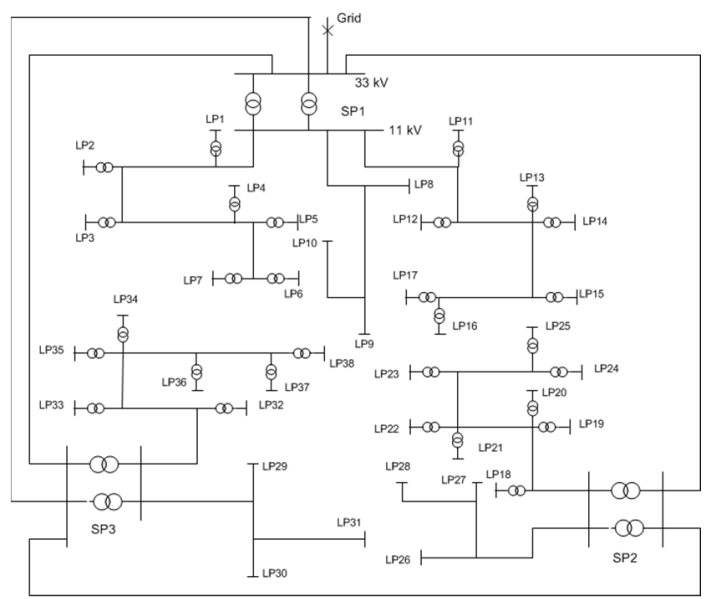

Fig. 2. Single Line Diagram of bus 4 Distribution System of RBTS [16]

Fig. 2 illustrates the single line diagram of the electric distribution system used in the case study. The electric distribution systems of the RBTS were designed following the general utility principles and practices regarding topology, ratings and load levels. They represent typical distribution networks. The bus 4 distribution system of the RTBS has a relatively complex topology and sufficient number of customers. Therefore, the bus 4 distribution system of the RTBS was chosen to carry out case studies. This medium voltage (MV) distribution network is comprised of three supply points (SPs) connected to the main grid by $33 \mathrm{kV} / 11 \mathrm{kV}$ transformers, 38 load points (LPs) and 7 feeders. The customer data are listed in Table I.

Table I

Customer Data

\begin{tabular}{|c|c|c|c|c|c|}
\hline \multirow{2}{*}{$\begin{array}{l}\text { Number } \\
\text { of Load } \\
\text { Points }\end{array}$} & \multirow{2}{*}{$\begin{array}{l}\text { Load } \\
\text { Points }\end{array}$} & \multirow{2}{*}{$\begin{array}{l}\text { Customer } \\
\text { Type }\end{array}$} & \multicolumn{2}{|c|}{$\begin{array}{l}\text { Load Level Per Load } \\
\text { Point (MW) }\end{array}$} & \multirow{2}{*}{$\begin{array}{l}\text { Number of } \\
\text { Customers }\end{array}$} \\
\hline & & & Average & Peak & \\
\hline 15 & $\begin{array}{l}1-4, \\
11-13, \\
18-21, \\
32-35\end{array}$ & Residential & 0.545 & 0.8869 & 200 \\
\hline 7 & $\begin{array}{ll}5, & 14, \\
15, & 22, \\
23, & 36, \\
37 & \\
\end{array}$ & Residential & 0.5 & 0.8137 & 200 \\
\hline 7 & $\begin{array}{l}8, \quad 10, \\
26-30\end{array}$ & Small User & 1.0 & 1.63 & 1 \\
\hline 2 & 9,31 & Small user & 1.5 & 2.445 & 1 \\
\hline 7 & $\begin{array}{lr}6, & 7, \\
16, & 17, \\
24, & 25, \\
38 & \end{array}$ & Commercial & 0.415 & 0.6714 & 10 \\
\hline Total & & & 24.58 & 40.00 & 4779 \\
\hline
\end{tabular}

The customer data consist of customer type, peak and average loads and number of customers. There are 4779 customers in total in the electric distribution network. The inverse demand function at each bus is assumed to be linear with a price elasticity of -0.1 . This level of demand price elasticity is con- 
sistent with empirical studies in [23]. There are 7 feeders in the electric distribution network. Each of the lines is one of the three types listed in Table II.

Table II

Connection Line Types

\begin{tabular}{|l|l|l|}
\hline $\begin{array}{l}\text { Connection } \\
\text { Line Type }\end{array}$ & $\begin{array}{l}\text { Line } \\
\text { Length } \\
(\mathrm{km})\end{array}$ & Line Number \\
\hline 1 & 0.6 & $\begin{array}{l}26101417212528303438414346 \\
49515558616467\end{array}$ \\
\hline 2 & 0.75 & $\begin{array}{l}14791216192224272932353740 \\
424548505356606365\end{array}$ \\
\hline 3 & 0.8 & $\begin{array}{l}358111315182023263133363944 \\
47525457596266\end{array}$ \\
\hline
\end{tabular}

\section{A. EV data}

A non-homogenous EV fleet is used for the EV charging management studies. The EV battery size varies according to individual $\mathrm{EV}$ driving requirements. It is assumed that the maximum charging power is $1.15 \mathrm{~kW}$ (based on a $5 \mathrm{~A}, 230 \mathrm{~V}$ connection). A typical value of $0.15 \mathrm{kWh} / \mathrm{km}$ is used to calculate the energy consumption while driving [24]. The minimum and maximum EV battery SOC is set as $20 \%$ and $85 \%$, respectively. The initial EV SOC varies by individual EV, and is set such that individual charging and driving requirements can be met. This is in accordance with the non-homogenous nature of EVs. A summary of the EV data is listed in Table III.

Table III

EV Data Summary

\begin{tabular}{|c|c|}
\hline EV Parameter & EV Parameter Value \\
\hline EV Battery Size & $25 \mathrm{kWh}$ \\
\hline Charging Power & $5.28 \mathrm{~kW}$ \\
\hline Energy Consumption of Driving & $150 \mathrm{Wh} / \mathrm{km}$ \\
\hline Minimum SOC & $20 \%$ \\
\hline Maximum SOC & $85 \%$ \\
\hline
\end{tabular}

\section{B. Driving data}

The Driving data used in the case studies are from the Danish National Travel Survey [24]. The Danish driving data were chosen for the case studies because the driving behavior in Denmark could be representative of the EV users' driving pattern. In Denmark, the average driving distance is about 40 $\mathrm{km}$ per day. Customers who need to drive a longer distance might not choose to use EVs.

The Danish driving data are highly detailed and provide significant insight into the driving habits of Danish residents. The relevant data used in this study are driving stop and start time, distance during driving periods, and day type. The EV availability for charging is defined as the periods during which the EV is parked. The driving profile from the same day type as the LMPs is used to create a more consistent test case. The EV availability on a working day is illustrated in Fig. 3. Each horizontal section represents a single EV, with the white colour representing availability to charge, and the black colour representing time periods when the EV is driving, and therefore is unavailable to charge.

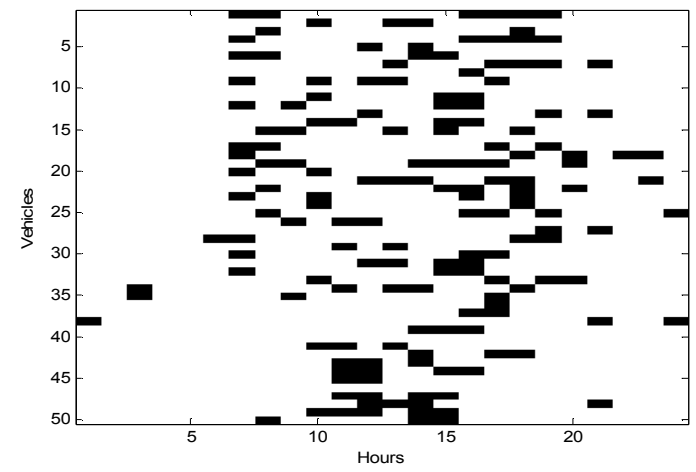

Fig. 3. EV Availability on a Working Day

\section{Case study results}

Three case studies listed in Table IV have been carried out. The EV penetration is defined as the ratio of maximum EV charging demand divided by the conventional household peak demand. The maximum EV charging demand is the sum of the EV charging demand when all EVs charge simultaneously.

Table IV

Case Study Scenarios

\begin{tabular}{|c|c|c|}
\hline Case Study No & Day Type & EV Penetration \\
\hline 1 & Tuesday & $100 \%$ \\
\hline 2 & Saturday & $100 \%$ \\
\hline 3 & Thursday & $500 \%$ \\
\hline
\end{tabular}

\section{Case Study 1}

The results of Case Study 1 are shown in Fig. 4-Fig. 6. Fig. 4 and Fig. 5 illustrate the effect of congestion alleviation on Line 1 when the DLMPs are introduced. Comparing with Fig. 4, the EV loads are spread out under the DLMPs in Fig. 5 and distributed among several hours with low LMPs, instead of charging all the EV loads in a single hour. In Fig. 6, the circles are the system LMP curve and the solid lines are the DLMPs at different nodes. The DLMPs are slightly higher than the system LMPs on the buses downstream to the congested line in order to shift away the EV loads to avoid severe congestion.

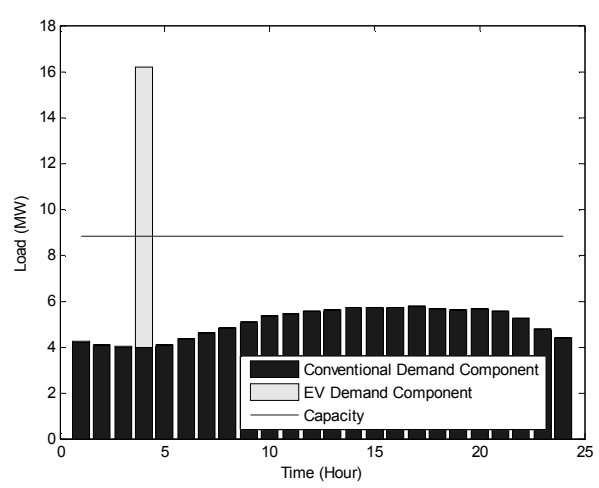

Fig. 4. Line 1 Loading without DLMPs of Case 1 


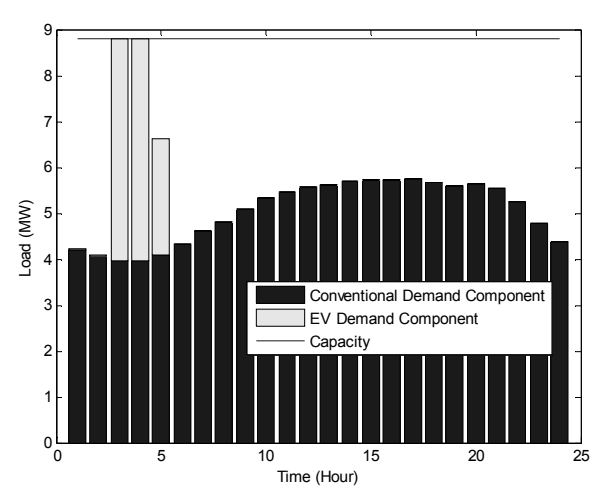

Fig. 5. Line 1 Loading with DLMPs of Case 1

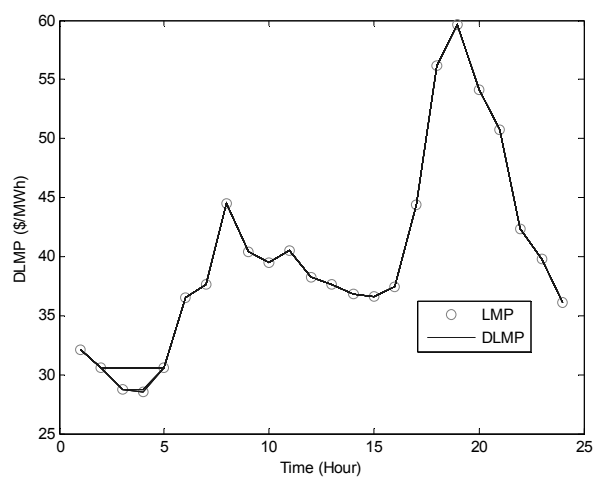

Fig. 6. System LMPs and DLMPs of Case 1

\section{Case Study 2}

The results of Case Study 2 are shown in Fig. 7-Fig. 9. In Case Study 2, the system LMP profile is different from the one in Case Study 1. The low system LMPs occur both in the morning and in the afternoon. Without the DLMPs, congestion occurs in both of the two periods on Line 1. With the proposed DLMP, it is shown in Fig. 8 that the congestion can be successfully alleviated. The EV loads have been shifted to the adjacent low LMP hours.

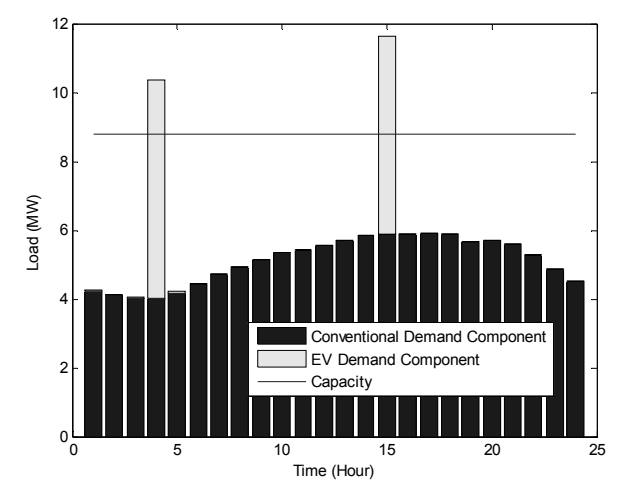

Fig. 7. Line 1 Loading without DLMP of Case 2

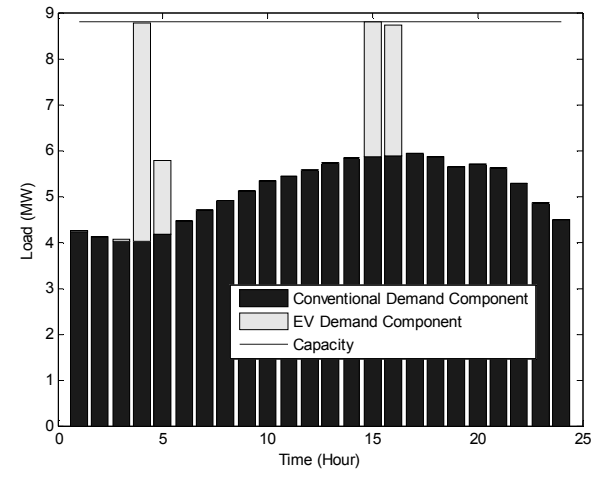

Fig. 8. Line 1 Loading with DLMP of Case 2

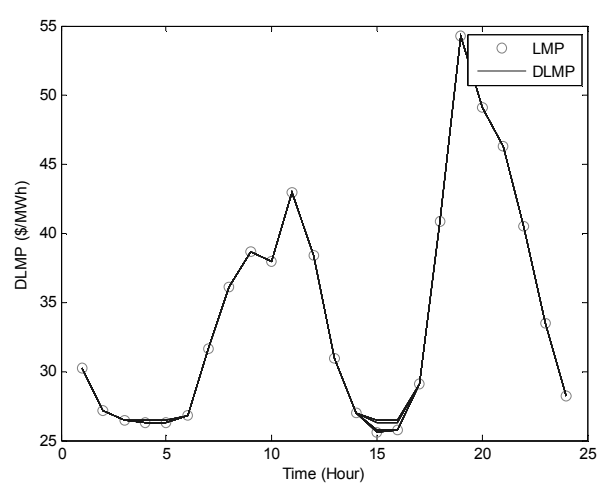

Fig. 9. System LMPs and DLMPs of Case 2

\section{Case Study 3}

In Case Studies 1 and 2, it is shown that DLMPs can alleviate the congestion induced by EVs under $100 \%$ EV penetration. In order to further illustrate the effectiveness of the proposed DLMP algorithm, studies with one projected future EV penetration levels have been conducted shown in Fig. 10 and Fig. 11 with $500 \%$ EV penetration.

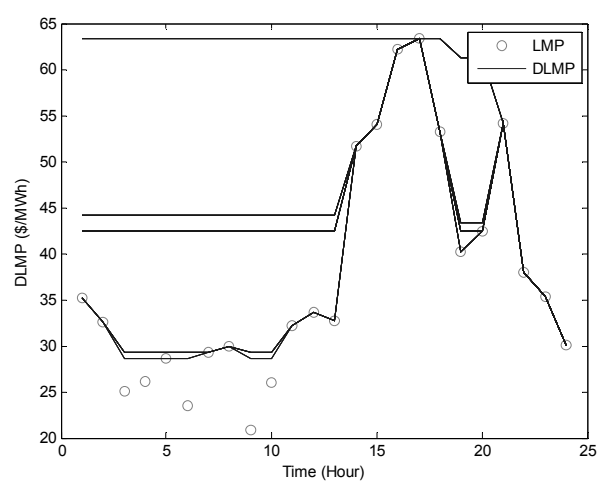

Fig. 10. DLMP with 500\% EV Penetration 


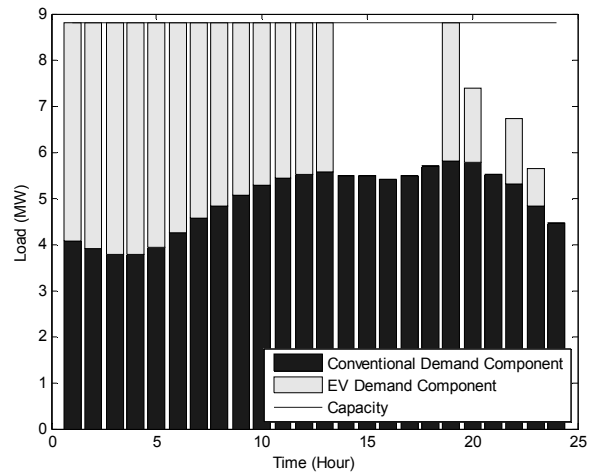

Fig. 11. Line 1 Loading with $500 \%$ EV Penetration

With 500\% EV penetration, the DLMPs are much higher than the system LMPs and the curve of DLMPs is flat in order to distribute the EV charging demand across time periods. Line capacity constraints are not violated shown in Fig. 11. From the Case Study 3 results presented, it can be concluded that the DLMP algorithm is a promising approach even with very high EV penetration, which is very likely to come into existence in the future.

\section{CONCLUSION}

An integrated DLMP algorithm has been proposed in order to handle the congestion within electric distribution networks faced by the future energy industry. The proposed DLMP algorithm optimizes social welfare to determine the DLMPs. These DLMPs can be used as price signals for EV aggregators to manage congestion within the electric distribution networks. Case studies with the RBTS electric distribution network and the Danish driving data have shown the efficacy of the proposed DLMP concept under the assumption that EV aggregators are price takers in the DSO market and under the used demand price elasticity. In a very extreme scenario with $500 \%$ EV penetration, the congestion in the electric distribution network can be alleviated by introducing the DLMPs. Future work will mainly cover the extension of existing framework to the environment where DSO only have imperfect information on the LMPs and use the forecast LMPs in decision-making.

\section{ACKNOWLEDGEMENT}

The work was funded by NSF Grant IIP 0969016, and by the members of the Power Systems Engineering Research Center (PSERC). The second author was supported by a fellowship from the Danish Agency for Science, Technology and Innovation (DASTI) during his research stay in the Department of Industrial Engineering and Operational Research (IEOR), UC Berkeley from Feb.-May 2012.

The authors would like to thank the Editor and the three anonymous reviewers for valuable comments that improved the paper and Peter F. Mayer from BC Hydro for proofreading the paper.

\section{REFERENCES}

[1] W. Kempton, and J. Tomic, "Vehicle-to-grid power fundamentals: calculating capacity and net revenue," J. Power Sources, vol. 144, no. 1, pp. 268-279, Jun. 2005.
[2] W. Kempton, and J. Tomic, "Vehicle-to-grid power implementation: from stabilizing the grid to supporting large-scale renewable energy, " $J$. Power Sources, vol. 144, no. 1, pp. 280-294, Jun. 2005.

[3] J. Tomic, and W. Kempton, "Using fleets of electric-drive vehicles for grid support," J. Power Sources, vol. 168, no. 2, pp. 459-468, Jun. 2007.

[4] E. Sortomme, and M. A. El-Sharkawi, "Optimal Charging Strategies for unidirectional vehicle-to-grid," IEEE Trans. Smart Grid, vol. 2. no. 1, pp. 131-138, March 2011.

[5] E. Sortomme, and M. A. El-Sharkawi, "Optimal Scheduling of Vehicleto-Grid Energy and Ancillary Services," IEEE Trans. Smart Grid, vol. 3. no. 1, pp. 351-359, Mar. 2012.

[6] G. T. Heydt, "The impact of electric vehicle deployment on load managment strategies," IEEE Trans. Power App. Syst., vol. 1, no. 144, pp. $1253-1259,1983$.

[7] K. Clement-Nyns, E. Haesen, and J. Driesen, "The impact of charging plug-in hybrid electric vehicles on a residential distribution grid," IEEE Trans. Power Syst., vol. 25, no. 1, pp. 371-380, Feb. 2010.

[8] K.J. Dyke, N. Schofield, and M. Barnes, "The impact of transport electrification on electrical networks," IEEE Trans. Ind. Electron., vol. 57, no. 12, pp. 3917-3926, Dec. 2010.

[9] J. A. Pecas Lopes, F. J. Soares, and P. M. Rocha Almeida, "Integration of electric vehicles in the electric power system," Proc. IEEE, vol. 99, no. 1, pp. 168-183, Jan. 2011.

[10] M. Arindam, S. K. Kyung, J. Taylor and A. Giumento, "Grid impacts of plug-in electric vehicles on Hydro Quebec's distribution system," in Proc. 2010 IEEE PES Transmission and Distribution Conference and Exposition, pp. 1-7.

[11] J. Taylor, M. Maitra, D. Alexander and M. Duvall, "Evaluation of the impact of plug-in electric vehicle loading on distribution system operations," in Proc. 2009 IEEE Power \& Energy Society General Meeting, pp. 1-6.

[12] O. Sundstrom, and C. Binding, "Flexible charging optimization for electric vehicles considering distribution grid constraints," IEEE Trans. Smart Grid, vol. 3. no. 1, pp. 26-37, Mar. 2012.

[13] N. Rotering, and M. Ilic, "Optimal charge control of plug-In hybrid electric vehicles in deregulated electricity markets," IEEE Trans. Power Syst., vol. 26, no. 3, pp. 1021- 1029, August 2010.

[14] R. D. Christie, B. F. Wollenberg and I. Wangensteen, "Transmission management in the deregulate environment," Proc. IEEE, vol. 88, no. 2, pp. 170-195, Feb. 2000.

[15] H. Glavitsch, and F. Alvarado, "Management of multiple congested congestions in unbounded operation of a power system," IEEE Trans. Power Syst., vol. 13, no. 3, pp. 1013-1019, Aug. 1998.

[16] R. N. Allan, R. Billinton, I. Sjarief, L. Goel and K. S. So, "A reliabiilty test system for educational purposes - basic distribution system data and results," IEEE Trans. Power Syst., vol. 6, no. 2, pp. 813-820, May 1991.

[17] S. Stoft, Power System Economics - Designing Markets for Electricity, New York: IEEE Press \& Wiley Interscience, 2002.

[18] F. Li, and R. Bo, "DCOPF-based LMP simulation: algorithm, comparison with ACOPF, and sensitivity," IEEE Trans. Power Syst., vol. 22, no. 4, pp. 1475-1485, Nov. 2007.

[19] J. Yang, F. Li and L. A. A. Freeman, "A market simulation program for the standard market design and generation/transmission planning," in Proc. 2003 IEEE Power Energy Society General Meeting, pp. 442-446.

[20] J. Yao, I. Adler, and S. Oren, "Modeling and computing two-settlement oligopolistic equilibrium in a congested electricity network," Operations Research, vol. 56, no. 1, pp. 34-47, January-February 2008.

[21] T. Limpaitoon, Y. Chen, and S. Oren, "The impact of carbon cap and trade regulation on congested electricity market equilibrium," Journal of Regulatory Economics, vol. 40, pp. 237-260, 2011.

[22] W. Hogan, "Contract networks for electric power transmission," Journal of Regulatory Economics, vol. 4, no. 3, pp. 211-242, 1992.

[23] I. L. Azevedo, M. G. Morgan, and L. Lave, "Residential and regional electricity consumption in the U.S. and EU: how much will higher prices reduce CO2emissions?" The Electricity Journal, vol. 24, no. 1, pp. 2129, 2011.

[24] Q. Wu, A. H. Nielsen, J. Østergaard, S.T. Cha, F. Marra, Y. Chen and C. Traeholt, "Driving pattern analysis for electric vehicle (EV) grid 
integration study," in Proc. Of 2010 IEEE PES Conference on Innovative Smart Grid Technologies Europe, pp. 1-6.

\section{BIOGRAPHIES}

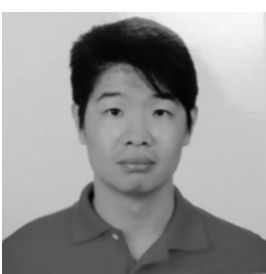

Ruoyang Li (S'13) received the B.S. degree in microelectronics from Fudan University in 2008, the M.S. degree in quantitative and computational finance from Georgia Institute of Technology in 2010, the M.A. degree in statistics from the University of California at Berkeley in 2011. He is currently pursuing the $\mathrm{PhD}$ degree in the Department of Industrial Engineering and Operations Research (IEOR) at the University of California at Berkeley. His research interests are optimization, applied statistics and economic modeling in energy markets.

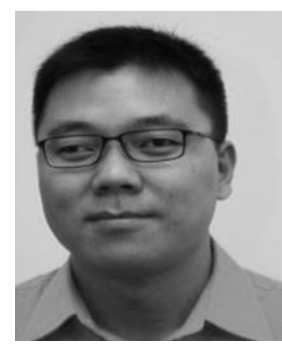

Qiuwei Wu (M'08) obtained the B. Eng. and M. Eng. from Nanjing University of Science and Technology, Nanjing, P. R. China, in 2000 and 2003, respectively, both in Power System and Automation. He obtained the $\mathrm{PhD}$ degree from Nanyang Technological University, Singapore, in 2009, in Power System Engineering.

He worked as a senior R\&D engineer in Vestas Technology R\&D Singapore Pte. Ltd. from Mar. 2008 to Oct. 2009. He joined Centre for Electric Technology (CET), Department of Electrical Engineering, Technical University of Denmark (DTU) as a PostDoc in Nov. 2009 and has been an assistant professor with CET since Nov. 2010.

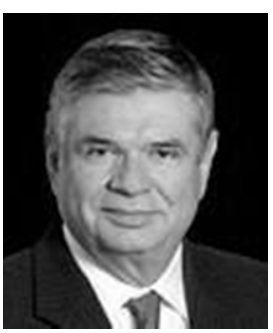

Shmuel S. Oren (F'02) received the B.Sc. and M.Sc. degrees in mechanical engineering and in materials engineering from the Technion Haifa, Israel, and the MS. and Ph.D. degrees in engineering economic systems from Stanford University, Stanford, CA, in 1972.

$\mathrm{He}$ is a Professor of IEOR at the University of California at Berkeley and the Berkeley site director of the Power System Engineering Research Center (PSERC). He is also member of the Market Surveillance Committee of the California ISO. He has published numerous articles on aspects of electricity market design and has been a consultant to various private and government organizations. Dr. Oren is a Fellow of INFORMS and of the IEEE. 